\title{
Physical parameters of components in close binary systems. I
}

\author{
J. M. Kreiner ${ }^{1}$, S. M. Rucinski ${ }^{2}$, S. Zola ${ }^{3}$, P. Niarchos ${ }^{4}$, W. Ogloza ${ }^{1}$, G. Stachowski ${ }^{1,5}$, A. Baran ${ }^{1,6}$, K. Gazeas ${ }^{4}$, \\ M. Drozdz ${ }^{1}$, B. Zakrzewski ${ }^{1}$, B. Pokrzywka ${ }^{1}$, D. Kjurkchieva ${ }^{7}$, and D. Marchev ${ }^{7}$ \\ 1 Mt. Suhora Observatory of the Pedagogical University, ul. Podchorążych 2, 30-084 Cracow, Poland \\ 2 David Dunlap Observatory, University of Toronto, PO Box 360, Richmond Hill, Ontario, L4C 4Y6, Canada \\ 3 Astronomical Observatory of the Jagiellonian University, ul. Orla 171, 30-244 Cracow, Poland \\ ${ }^{4}$ Department of Astrophysics, Astronomy and Mechanics, Faculty of Physics, University of Athens, Panepistimiopolis, \\ 15784 Zografos, Athens, Greece \\ 5 N. Copernicus Astronomical Center, ul. Bartycka 18, 00-716 Warsaw, Poland \\ 6 Torun Centre for Astronomy, ul. Gagarina 11, 87-100 Torun, Poland \\ 7 Department of Physics, Shoumen University, 9700 Shoumen, Bulgaria
}

Received 19 June 2003 / Accepted 12 September 2003

\begin{abstract}
The paper presents combined spectroscopic and photometric orbital solutions for four close binary systems: SW Lyn, QW Gem, AP Leo and V2150 Cyg. The photometric data are new, while the spectroscopy has been recently obtained within the radial velocity programme at the David Dunlap Observatory. This paper is the first in the planned series of investigations. We give an extensive description of the motivation for the series and of the main assumptions made in our solutions. The four targets of this investigation span a range of typical configurations and thus present different levels of difficulty for the combined spectroscopic and photometric orbital solutions.
\end{abstract}

Key words. binaries: eclipsing - binaries: close - binaries: contact - stars: fundamental parameters

\section{Introduction}

W UMa-type contact binary stars are surprisingly common in the solar neighbourhood. Although there has long been an awareness of their large number relative to other types of variables, it was not until large samples provided by microlensing surveys such as OGLE (Rucinski 1998) became available that the first reliable estimates of their spatial frequency were made. The apparent relative frequency of W UMa-type stars was estimated at about $1 / 130$ of the frequency of the common solar-neighbourhood FGK-type disc population dwarfs with an age of $\simeq(5-11) \times 10^{9}$ years. The real, still higher space density remains unknown, as it is closely linked to the unknown distribution of mass ratios (cf. Rucinski 1997). Recently, Rucinski (2002a) re-analysed the Hipparcos sample and found that the statistically small but very well defined sample of nearby systems suggests a somewhat lower frequency of about 1/500; the OGLE direction passing close to the Galactic Centre may show a much higher frequency than in the solar neighbourhood volume. The frequency rises with the age of the system, so that open clusters have relatively fewer of them than the typical disc population, which can reach an age of 11 billion years (Binney et al. 2000). W UMa-type systems are not observed in clusters younger than $0.7 \times 10^{9}$ years. This is in exact agreement

Send offprint requests to: J. M. Kreiner,

e-mail: sfkreine@cyf-kr.edu.pl with theoretical models, which describe the formation of contact systems by the gradual loss of angular momentum due to the combined effects of tidal coupling and braking by the stellar wind (Vilhu 1981, 1982; Rahunen 1981, 1982, 1983).

However, the details of the evolution remain unknown, as we do not know what happens at the moment when the contact binary is formed. We do know that, on a very short (dynamical) time scale, a new object is formed with a new mass ratio $q_{\mathrm{c}}$, which is probably smaller than before contact $\left(q_{\mathrm{c}}<q_{\mathrm{pc}}\right)$, but we do not usually know its value. The newly-formed structure is to some extent simpler (one object whose surface is described by one gravitational potential), but deep inside - and so invisible - simultaneous energy and mass transport processes occur: energy flows from the more massive component to the less massive component, mass in the opposite direction. These fascinating processes are very poorly understood, as is the speed with which the system evolves and why it remains in contact. The so-called thermal relaxation oscillation model predicts an oscillatory reconnection and breaking of the contact. Simple estimates of the global evolution linked to momentum loss suggest that for small $q$ this process, which takes place roughly in the thermal timescale of the secondary component, becomes as slow as that of the nuclear evolution of the more massive component, i.e. of the order of several Gyr. This must lead to a slow accumulation of contact systems with $q_{\mathrm{c}} \rightarrow 0$. Before this happens, however, a new dramatic dynamical process at 
$q \approx 0.09$ (Webbink 1976; Rasio 1995) suddenly changes the contact system into a single, rapidly rotating star which loses angular momentum through the stellar wind resulting from an unusually high level of solar-type activity. The binary system ceases to exist. As a result, thanks to the extremely slow evolution at $q_{\mathrm{c}} \approx 0.09$ (a timescale can be as long as $10^{11}$ years), one can expect the accumulation of a very large number of systems just above $q_{\mathrm{c}}$. The above description is confirmed by the high frequency of contact binary systems, particularly in respect to the slower evolution at $q_{\mathrm{c}}$. Unfortunately a true understanding of the evolution is still far off.

The ease of observation of W UMa systems has meant that they have been the subject of many poorly-planned programmes (particularly in terms of the choice of targets), which has led to a lack of significant results despite many observations. This work aims to address these issues by carefully choosing target objects so as to maximise their usefulness for understanding the evolution of contact binaries. In addition to a better understanding of the W UMa-type systems, the results of our project can also be used for the study of the structure of our Galaxy, the clusters and nearby galaxies.

Rucinski (1994) pointed out the simple, empirical calibration of absolute brightness as a function of the orbital period and any parameters dependent on $T_{\text {eff }}$, for example: the colour index: $M_{V}=M_{V}(\log P,(B-V))$. This empirical relationship can be used to verify the membership of a particular system in open and even globular clusters, which in turn could help to determine the origin of the blue stragglers, among which the true (spatial) frequencies of W UMa systems may be as high as $1 / 30$ - i.e. several times higher than that of the old disc population (Rucinski 2000). W UMa-type systems could thus play the same role for the disc population as pulsating RR Lyr stars do for the halo population - and there are about $7 \times 10^{4}$ times as many W UMa-type stars per unit volume in the solar neighbourhood.

The use of the $M_{V}$ brightness calibration does, however, have its weak points. W UMa-type systems have photospheric spots, which make the calibration more difficult. Photometric observations therefore have to be repeated to follow the evolution of the spots, but some scatter in the calibration is very difficult to avoid. Also, the calibration in its current form does not take into account the expected influence of the mass ratio on $M_{V}$. This can only be determined using systems which have a full photometric and spectroscopic description. One of the most important aims of this project is to determine the dependence $M_{V}=M_{V}(q)$ by combining spectroscopy and photometry.

\section{Selection of the objects}

The spectroscopic program of radial velocity measurements has been conducted since 1999: Lu \& Rucinski (1999), Rucinski \& Lu (1999), Rucinski et al. (2000), Lu et al. (2001), Rucinski et al. (2001), Rucinski et al. (2002). So far, it has resulted in 70 new radial velocity orbits, all determined with the same instrument, using the same methods and suffering from the same uncertainties of the order of $1-2 \mathrm{~km} \mathrm{~s}^{-1}$. Selection of the targets has been quasi-random: within each season, a few dozen close binary systems with periods shorter than one day, brighter than $11 \mathrm{mag}$ and with declinations $>-20^{\circ}$ are observed and then the results are published in groups of ten systems as soon as reasonable orbital elements are obtained from measurements evenly distributed in orbital phase. For technical details and conventions, and for preliminary estimates of errors and uncertainties, see the interim summary paper by Rucinski (2002b).

Although all available short-period binaries are observed, our list is dominated by W UMa type systems for reasons as we described above. For these binaries, we are almost always able to determine the velocities of both components. The ratio of the semi-amplitudes, $K_{2} / K_{1}$, even for very simple, preliminary sine fits, gives a very well determined value of the mass-ratio, $q$. This physically crucial parameter is exceedingly difficult to determine from photometric observations and the literature is full of photometric determinations which were later disproved when spectroscopic observations became available. While the values of $q_{\mathrm{sp}}$ are well determined, the individual values of $K_{i}$ may have systematic biases when sine fits are used; this obviously results in biased determinations of the most important final parameters which are the stellar masses. These can be done only when the orbital inclination is known, but this in turn requires detailed analysis of the light curves. Thus, the optimum approach is to use the spectroscopic values of the mass ratio for full, combined photometric/spectroscopic solutions.

In this first of the series of papers, we present results for four systems (SW Lyn, QW Gem, AP Leo and V2150 Cyg), as well as the method for deriving accurate absolute parameters of the components from light curve modelling combined with spectroscopic data. The objects have been selected in view of the natural grouping within the class of considered objects. Based on the shape of the light curve, the objects in our sample can be divided into four groups: near contact systems, contact systems without noticeable spots, contact systems with spotted light curves and ellipsoidal systems with amplitude of light variations being of order of $0.1 \mathrm{mag}$ or less. In this paper we present results for four systems representing each of the four groups in our sample.

Papers presenting the parameters of other systems from our sample will follow as soon as new photometric as well as spectroscopic observations and computations are completed.

\section{New photometric data}

For most of the systems in our sample we decided to gather new, multicolour light curves taken with CCD's or a PMT photometer. For this project, observations at Mt. Suhora Observatory are being done using the $60 \mathrm{~cm}$ telescope equipped with 2- or 3-channel photometers with Hamamatsu R-2949 photomultipliers. A PMT-based photometer at the $75 \mathrm{~cm}$ telescope has also been used at the South African Astronomical Observatory in Sutherland for targets with negative declination. Both photometers are equipped with a set of wideband Johnson-Morgan filters. In the other observatories (the $40 \mathrm{~cm}$ telescope at Athens University, the $1.2 \mathrm{~m}$ telescope at the Kryonerion Observatory, the $50 \mathrm{~cm}$ telescope at the Astronomical Observatory of the Jagiellonian University and the $50 \mathrm{~cm}$ telescope at the Skibotn Observatory, University of 
Table 1. Linear elements used for phasing observations.

\begin{tabular}{lcl}
\hline \hline star & reference epoch $\left(\mathrm{JD}_{\text {hel }}\right)$ & period (days) \\
\hline SW Lyn & 2450753.5418 & 0.644066253 \\
QW Gem & 2451927.5723 & 0.358127 \\
AP Leo & 2452321.5705 & 0.43035716 \\
V2150 Cyg & 2452477.4250 & 0.591856 \\
\hline
\end{tabular}

Troms $\varnothing$ ) observations are done using CCD photometers also equipped with a set of wide-band filters. Over the scope of this program the complete light curves of all targets will be gathered only using one instrument with the aim of achieving the best accuracy by avoiding calibration problems when combining observations from different observatories.

All new light curves reported in this paper have been gathered at the Mt. Suhora Observatory using the two channel photometer. A more detailed equipment description can be found in Kreiner et al. (1993). We tried to gather the complete light curve of each system in as short as possible time in four $(B V R I)$, wide band filters. Observations of SW Lyn were obtained over 5 nights: 31 Oct./1 Nov. 1997, 19/20, 26/27, 27/28 Jan. and 8/9 Feb. 1998. A complete light curve for QW Gem was gathered during only one night, 17/18 Jan. 2001. AP Leo was observed during 5 nights: 15/16 Feb., 10/11, 11/12, 13/14 and 30/31 March 2001. Four nights were needed to obtain the light curve of V2150 Cyg: 21/22, 26/27, 27/28 and 28/29 July 2002. The data were first cross-calibrated for differential channel amplification, next corrected for differential extinction using the mean extinction coefficients for the observatory. The data were left in the instrumental system, but for the light curve modelling we recalculated magnitude differences between the target and comparison stars into flux units. The observations were phased using the time of the most recently observed primary minimum as the reference epoch, with a linear ephemeris for each target system and the period usually taken from Kreiner et al. (2001). This catalogue is available on-line at the following address: http://www .as.ap.krakow.pl/o-c/index.php3. For the systems discovered by the Hipparcos mission, we also used the time of the primary minimum from the new data, but the Hipparcos period to phase the observations. The reference epochs and periods we used are listed in Table 1.

\section{Light curve modelling}

\subsection{The method for deriving global parameters}

For each system we fix two crucial parameters, the temperature and the mass ratio, as derived from spectroscopy: the temperature will always be assumed as that appropriate for the spectral type (Harmanec 1988) and the mass ratio will be that derived from the radial velocity curves. Such an approach allows derivation of the physical parameters reliably without the danger of getting spurious values, often seen in solutions for contact systems where fits of almost the same quality have been achieved for a large range of mass ratio values, thus resulting in absolute parameters of components which differ significantly.

In order to obtain the physical parameters we used the Wilson-Devinney code (W-D) (Wilson \& Devinney 1973;
Wilson 1979, 1993) which has now become a standard tool. However, due to complicated light changes (caused, for example, by surface spots or a third star) seen in many systems, which make the solutions very difficult or non-unique with methods seeking only a local minimum, we decided to apply a Monte Carlo search. First, this algorithm searches for a global minimum in a given range of parameters and, second, it is independent of correlations between parameters describing the system. The second property is especially important for systems with a third light which is usually correlated with many parameters. We also apply this method to systems for which the configuration is uncertain or the light curve is distorted by the presence of the spots. In the first case, the Monte Carlo search does not require a priori assumption of a system configuration - it will be found after computations have converged. If a spot is included in the model then additional free parameters (four for each spot) are required, some of them heavily correlated to each other thus making the location of the global minimum very difficult.

The search for the best fit was done in the following way: the search array of size of either 1000 (systems without spots) or 2000 elements (AP Leo) was first filled with randomly generated parameters in chosen ranges. Each element of the array consists of the whole set of parameters needed to generate a theoretical light curve. Next, after each trial, the element with the largest $\chi^{2}$ was replaced by a new one, if the fit was better (measured by the $\chi^{2}$ ). The whole procedure was repeated, until convergence was achieved, i.e. the difference between elements with largest and smallest $\chi^{2}$ values in the search array was small.

\subsection{SW Lyn}

Recent photometric and period study of this system was done by Ogloza et al. (1998). The light curve modelling of the $B$ and $V$ light curves gave the best fit for a mass ratio in the range 0.5-0.6. However, the models for this star were proposed for a mass ratio fixed at $q=0.35$ as derived from the spectroscopic observations by Vetesnik (1977). The study of the difference between observed and calculated times of minima $(\mathrm{O}-\mathrm{C})$ revealed that the $\mathrm{O}-\mathrm{C}$ diagram was modulated, due to presence of a third component, with a periodicity of 2128 days. The new radial velocity curves were obtained by Lu et al. (2001) and they were entirely different from that of Vetesnik (1977). No apparent eccentricity was found and the new mass ratio of SW Lyn was found to be $q=0.52 \pm 0.03$. The presence of a third component was confirmed and it was found to be about three times fainter than the binary system. The difference in the RV results can be explained by the inadequate spectral resolution of Vetesnik's observations.

Due to the above complications we applied a Monte Carlo search to get the best solution for the new BVRI light curves. We fixed the mass ratio at $q=0.52$ and the temperature of the primary at $6700 \mathrm{~K}$ as corresponding to its spectral type (F2V Lu et al. 2001). Due to the presence of the third component, we included a third light into the list of free parameters. The third light contribution to the total light of the system was 
Table 2. Results derived from the light curve modelling.

\begin{tabular}{lrrrr}
\hline \hline parameter & SW Lyn & QW Gem & AP Leo & V2150 Cyg \\
\hline configuration & near-contact & contact & contact & contact \\
filling factor & & $23 \%$ & $6 \%$ & $19 \%$ \\
phase shift & $0.0004 \pm 0.0001$ & $-0.0006 \pm 0.0003$ & $0.0011 \pm 0.0003$ & $0.0152 \pm 0.0003$ \\
$i$ (degrees) & $88.07 \pm 0.66$ & $80.45 \pm 0.22$ & $78.00 \pm 0.31$ & $43.39 \pm 0.84$ \\
$T_{1}(\mathrm{~K})$ & $* 6700$ & $* 6100$ & $* 6150$ & $* 8000$ \\
$T_{2}(\mathrm{~K})$ & $4480 \pm 12$ & $5890 \pm 10$ & $6250 \pm 25$ & $7920 \pm 42$ \\
$\Omega_{1}$ & $3.065 \pm 0.0029$ & $6.469 \pm 0.0036$ & $2.434 \pm 0.0032$ & $3.318 \pm 0.0101$ \\
$\Omega_{2}$ & $3.119 \pm 0.017$ & $* * 6.469$ & $* * 2.434$ & $* * 3.318$ \\
$\left.q m_{2} / m_{1}\right)$ & $* 0.524$ & $* 2.994$ & $* 0.297$ & $* 0.802$ \\
\hline$L_{1}^{s}(B)$ & $10.145 \pm 0.151$ & $3.185 \pm 0.015$ & $9.200 \pm 0.053$ & $5.593 \pm 0.181$ \\
$L_{1}^{s}(V)$ & $9.658 \pm 0.146$ & $2.811 \pm 0.013$ & $9.238 \pm 0.048$ & $4.785 \pm 0.150$ \\
$L_{1}^{s}(R)$ & $9.353 \pm 0.144$ & $2.609 \pm 0.013$ & $9.306 \pm 0.044$ & $4.872 \pm 0.151$ \\
$L_{1}^{s}(I)$ & $8.893 \pm 0.137$ & $2.225 \pm 0.017$ & $9.385 \pm 0.039$ & $4.668 \pm 0.146$ \\
$L_{2}^{s}(B)$ & $* * 0.575$ & $* * 7.224$ & $* * 3.168$ & $* * 4.404$ \\
$L_{2}^{s}(V)$ & $* * 0.703$ & $* * 6.471$ & $* * 3.152$ & $* * 3.795$ \\
$L_{2}^{s}(R)$ & $* * 0.832$ & $* * 6.113$ & $* * 3.147$ & $* * 3.883$ \\
$L_{2}^{s}(I)$ & $* * 1.049$ & $* * 5.357$ & $* * 3.129$ & $* * 3.745$ \\
$l_{3}(B)$ & $0.131 \pm 0.011$ & $0.148 \pm 0.004$ & $0.004 \pm 0.003$ & $0.061 \pm 0.028$ \\
$l_{3}(V)$ & $0.152 \pm 0.011$ & $0.240 \pm 0.003$ & $0.006 \pm 0.002$ & $0.201 \pm 0.024$ \\
$l_{3}(R)$ & $0.174 \pm 0.011$ & $0.285 \pm 0.003$ & $0.000 \pm 0.001$ & $0.197 \pm 0.024$ \\
$l_{3}(I)$ & $0.183 \pm 0.010$ & $0.374 \pm 0.004$ & $0.000 \pm 0.001$ & $0.231 \pm 0.023$ \\
\hline$r_{2}{ }^{\text {pole }}$ & $0.2735 \pm 0.0024$ & $0.4567 \pm 0.0003$ & $0.2640 \pm 0.0028$ & $0.3528 \pm 0.0015$ \\
$r_{1}{ }^{\text {point }}$ & $0.4597 \pm 0.0011$ & & & \\
$r_{2}{ }^{\text {point }}$ & $0.3159 \pm 0.0046$ & & & \\
$r_{1}$ side & $0.4076 \pm 0.0006$ & $0.2920 \pm 0.0004$ & $0.4974 \pm 0.0010$ & $0.4132 \pm 0.0019$ \\
$r_{2}{ }^{\text {side }}$ & $0.2821 \pm 0.0027$ & $0.4920 \pm 0.0004$ & $0.2754 \pm 0.0009$ & $0.3723 \pm 0.0019$ \\
$r_{1}$ back & $0.4284 \pm 0.0007$ & $0.3328 \pm 0.0006$ & $0.5238 \pm 0.0012$ & $0.4509 \pm 0.0017$ \\
$r_{2}{ }^{\text {back }}$ & $0.3007 \pm 0.0034$ & $0.5212 \pm 0.0014$ & $0.3115 \pm 0.0015$ & $0.4130 \pm 0.0029$ \\
\hline$C^{s}$ ( & & & &
\end{tabular}

$*-$ not adjusted, $* *$-computed, $L_{1}^{s}, L_{2}^{s}$ : W-D program input values.

The subscripts 1 and 2 refer to the star being eclipsed at primary and secondary minimum, respectively.

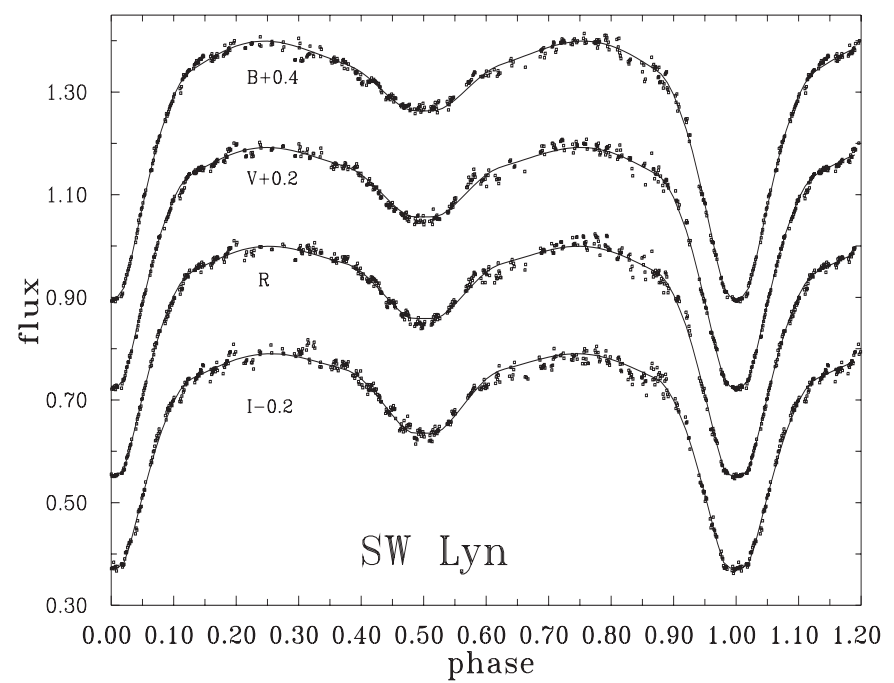

Fig. 1. Comparison between theoretical and observed light curves of SW Lyn ( $B V R I$ filters). Individual observations are shown by squares and theoretical curves by lines.

searched in the range between 0 to $50 \%$. We set the following ranges for the free parameters: the phase shift between -0.01 and 0.01 , inclination between 60 and 90 degrees, temperature of the secondary between $4000 \mathrm{~K}$ and $6700 \mathrm{~K}$, the components potentials between 1.8 and 8.0 and the luminosity of primary between 1 and 13. The theoretical values of both albedo and gravity darkening coefficients were used. The limb darkening coefficients were adopted as functions of the temperature and wavelength from Díaz-Cordovés et al. (1995) and Claret et al. (1995) tables. Furthermore, we set the dimension of the search array to have 2000 elements. We proceed with computations until the difference between the best and the worst elements in the search array was less than 1 percent. The results are presented in Table 2 and comparison between observed and synthetic light curves is shown in Fig. 1. All of the variable parameters have been given with errors.

\section{3. $Q W$ Gem}

This system was discovered by Hipparcos. The first radial velocity orbital solution was made by Rucinski et al. (2003) who estimated its spectral type to be F8V. They also classified this contact system as a W-type. The mass ratio given by Rucinski et al. (2003) is $0.334 \pm 0.009$. Since QW Gem is a W-type contact system, the mass ratio used in the light curve modelling is $1 / q$ due to the definition of components required by the W-D code.

New photometric observations were made with $B, V, R$ and $I$ filters, about 400 single points in each filter. The light curve 


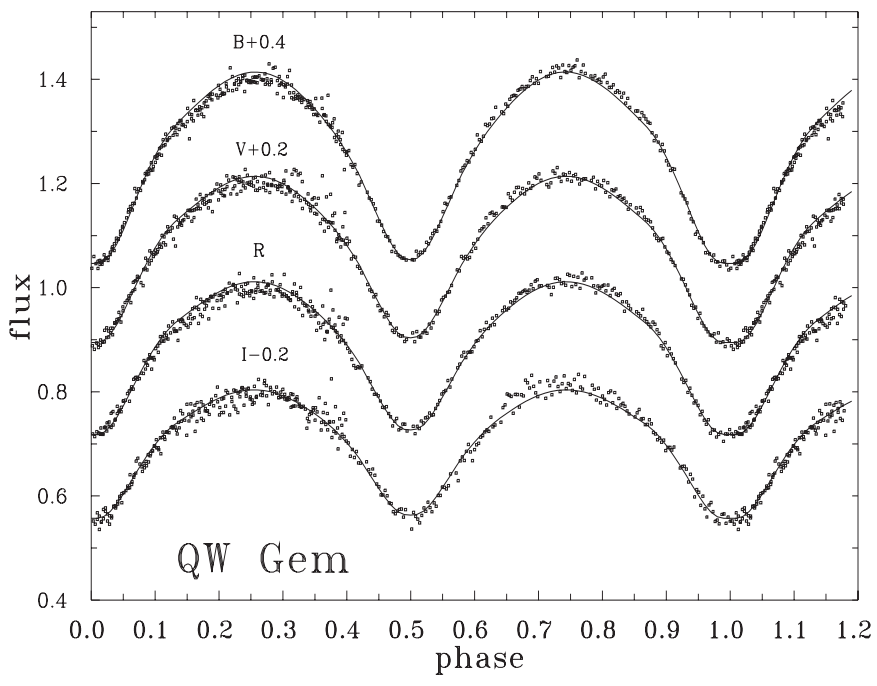

Fig. 2. Comparison between theoretical and observed light curves of QW Gem (BVRI filters). Individual observations are shown by squares and theoretical curves by lines.

of this system looks relatively uncomplicated, only a very small O'Connell effect (a difference in the height of the maxima) is visible. Therefore, for the initial solution, we applied the differential correction search method of the W-D code. The mass ratio given by the orbital solution and the temperature of the primary $\left(T_{1}=6100 \mathrm{~K}\right)$ according to the spectral type of the system (Rucinski et al. 2003) were fixed. The system configuration was set to be contact (mode 3 ). The phase shift, inclination, the temperature of the secondary star, the potential and the luminosity of the primary component were varied. Additionally, we also assumed that there is no third light in the system. It turned out that no good fit to the observations can be obtained under the above assumptions. As noted by Rucinski et al. (2003) the Hipparcos parallax of QW Gem has a large error, which might indicate some undiscovered companion. We added third light as a free parameter and therefore we also switched to the Monte Carlo search to avoid problems with finding the best solutions due to the presence of the third light, which is heavily correlated with some parameters, especially the luminosities of the components. This time a reasonably good fit was obtained quickly and the results are presented in Table 1 (system parameters), while the comparison between observations and our theoretical light curve is shown in Fig. 2.

\subsection{AP Leo}

The eclipsing system AP Leo has been investigated most often among the systems we analysed in this paper. Its variability was discovered by Strohmeier \& Knigge (1961) who marked it as BV366. The first photoelectric light curves were obtained by Mauder (1967) and Bartolini \& Battistini (1969). Discussion of period and light curve changes was recently published by $\mathrm{Li}$ et al. (2002). The spectroscopic orbit of this system was determined by Rucinski \& Lu (1999) for the first time giving the mass ratio $q=0.297 \pm 0.009$. They estimated spectral type as $\mathrm{F} 7-8 \mathrm{~V}$ and the temperature corresponding to this type was assumed in the light curve modelling.

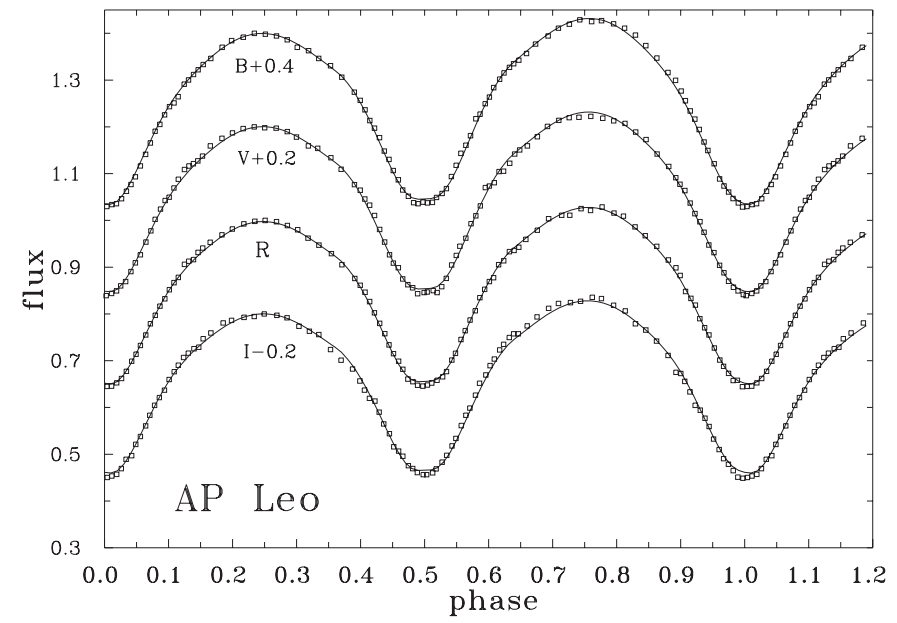

Fig. 3. Comparison between theoretical and observed light curves of AP Leo ( $B V R I$ filters). Individual observations are shown by squares and theoretical curves by lines.

The new, BVRI light curves of AP Leo reveal heavy distortions, most often explained as being due to the presence of spots. Since our goal is to derive accurate parameters of components we decided to get rid of disturbances of the light curve as accurately as possible. Therefore, we applied the Monte Carlo search, including one cool spot in the model. We searched for the spot location on the whole surface of the primary and ranges for the spot size and temperature were: (10-70 degrees) and 0.5 to 1.0 , respectively. We also included a third light into the list of free parameters as suggested by Zhang et al. (1992). Other free parameters were: phase shift, the system inclination $(i)$, the secondary component temperature $\left(T_{2}\right)$, the potentials of both components $\left(\Omega_{1,2}\right)$ and the luminosity of the primary star $\left(L_{1}\right)$. Two separate computations were performed: one assuming the system to be an A-type and the other assuming a W-type. The parameters giving the best fit are presented in Table 2. We obtained better description of the new observations under assumption that AP Leo is an A-type contact system. Our solution converged in a negligible amount of the third light which supports the result by Rucinski \& Lu (1999). The theoretical versus observed light curves are shown in Fig. 3.

\subsection{V2150 Cyg}

V2150 Cyg is another new binary system discovered by the Hipparcos mission. The orbital period is 0.591 days. Abt (1981) classified its spectral type to be A5V. The Hipparcos light curve shows variations only slightly above $0.1 \mathrm{mag}$ indicating a low orbital inclination. The variable system is a member of the ADS 14835 visual binary. The companion is faint (3.4 mag) at the distance of about 4 arcsec from the close binary. Lu et al. (2001) estimated the spectral type of the star to be $\mathrm{A} 6 \mathrm{~V}$ and published the radial velocity curves of both components. The mass ratio was found to be $q=0.802 \pm 0.006$, with the more massive, hotter component being eclipsed at the primary minimum. As noted by Lu et al. (2001) this system is important since it can provide an extension of the absolute magnitude calibration toward early spectral types. 


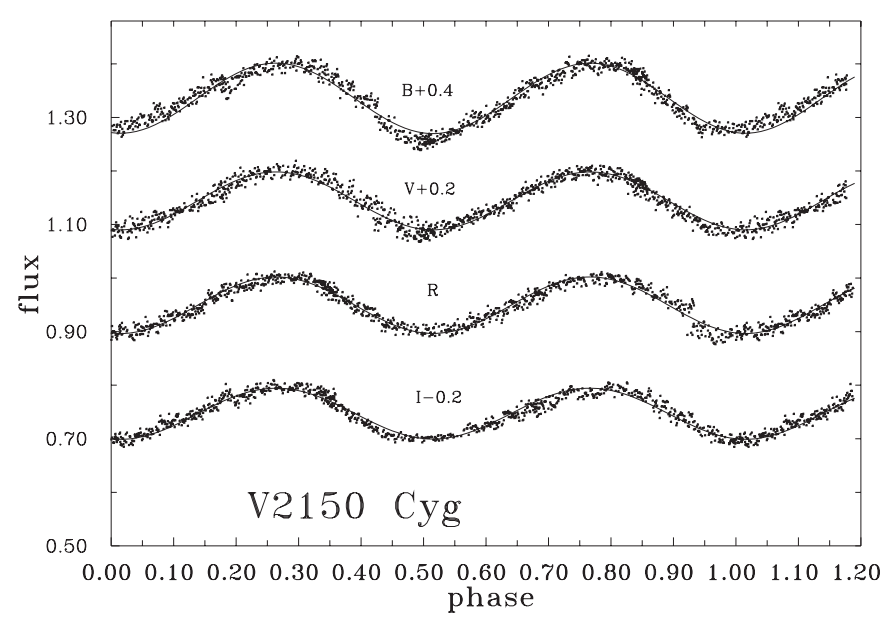

Fig. 4. Comparison between theoretical and observed light curves of V2150 Cyg (BVRI filters). Individual observations are shown by squares while lines represent the theoretical light curves.

Since much information is obtained from observations of the eclipses, we expect that the quality of determination of the absolute parameters of the system will not be as good as for systems with higher inclination. Therefore, for such systems we have applied the Monte Carlo search method, especially if there is also a spectroscopically detectable third star or an indication of its light contribution in the photometric data. Apart from fixing the mass ratio at the value from the spectroscopy and the temperature of the primary to that corresponding to the A6V spectral type ( $\mathrm{Lu}$ et al. 2001), we set the following ranges for the free parameters: phase shift $(-0.02-0.02)$, inclination (10-90 degrees), temperature of the secondary component $(4000-8000 \mathrm{~K})$, potentials of the components $(1.5-5.0)$, the luminosity of the primary (1-13) and the third light contribution (0-0.3).

Although convergence was also obtained for this noneclipsing system, the fit is not as good as for the three other systems as it can be seen in Fig. 4. The system parameters are presented in Table 2.

\section{Discussion}

We present results of the combined photometric/spectroscopic solution of four W UMa type systems selected in order to illustrate the cases most commonly encountered when combining photometry and spectroscopy to derive physical parameters of close binary systems. The solutions utilize new photometric data obtained through an international collaboration and the availability of new, homogeneous spectroscopic data from the David Dunlap Observatory. Of particular importance are the spectroscopic values of the mass ratio $q_{\mathrm{sp}}$ which were kept fixed in the light curve analysis, thus helping to improve the quality of our final combined solutions. This paper is the first in a series which will lead to a systematic rediscussion of several short-period binary stars.

We also present in detail the methods used to obtain the parameters of the components as accurately as possible for our sample of close binary systems. The fixing of the two crucial parameters as known from spectroscopy leads to fast and reliable solutions of the light curves of those systems having 'undisturbed' light curves. If there are no additional components in a binary system, then the differential correction algorithm is the fastest way to obtain the results. We anticipated such a situation for QW Gem after having seen its light curve, however, during the computation process, it turned out that for this specific system a good fit cannot be obtained without a third light present in the solution. We note the necessity of using the Monte Carlo search procedure for very close (nearcontact) or minimal degree contact systems. This algorithm does not require choosing a priori the geometry of the system by fixing the mode parameter in the Wilson-Devinney code, since it searches for a best fit in a given range of the potentials of components. SW Lyn is an example of such a system where a semidetached configuration with either primary (mode 4) or secondary (mode 5) filling their Roche lobes gave comparable results (Ogloza et al. 1998).

For systems which either have a third light or which have light curves affected by spots, we also use the Monte Carlo search algorithm for solving the light curves, as it works even if some (or many) parameters are correlated. We emphasise that even if a spot or spots are included in our computations (one cool spot in the case of AP Leo in this paper), we include them solely for the purpose of removing observed distortions in the light curves and in order to obtain reliable physical parameters. Most of the difficulties encountered were with the fourth system analysed, V2150 Cyg. This is for two reasons: first, this is a non-eclipsing system where information about its geometry comes only from the ellipticity effects and, second, the light curves are distorted in a peculiar way: the decrease toward the secondary minimum is much steeper than the rising branch, somewhat resembling that of a pulsating star. In addition, the system has a close companion star (a third light), which was within the photometer aperture during measurements. Inclusion of the third light further complicated the solution of the light curve.

The errors shown in Table 1 and subsequently used for calculating the errors of the absolute parameters were obtained from the Monte Carlo computations. For each light curve, normal points were calculated, around 100 of them in each filter. Each normal point was assigned a standard error calculated from all individual contributing observations. The desired confidence level was determined as a function of number of free parameters used in the $\chi^{2}$ minimization (Numerical Recipes in Fortran, 2nd ed., Sect. 15.6). Since in our case number of free parameters exceeded 6, as given in the book, we used linear extrapolation for the larger number of free parameters (in our case 12-14), obtain the $\chi^{2}$ limits for the confidence level of $90 \%$. During the computations, the $\chi^{2}$ value was monitored and when the condition of 90 percent confidence level was fulfilled, the search array was saved and standard values of sigma were later calculated for each free parameter from all elements stored in the array. The use of the more generous confidence level of 90 percent (in place of the usual 67 percent, as for the standard mean error), as well as allowance for the multiparametric broadening of the confidence levels, gives us assurance that our errors are correctly estimated and - if anything 
Table 3. Absolute parameters of the studied systems (in solar units).

\begin{tabular}{lcccc}
\hline \hline system & $\mathcal{M}_{1}$ & $\mathcal{M}_{2}$ & $R_{1}$ & $R_{2}$ \\
\hline SW Lyn & $1.716 \pm 0.055$ & $0.899 \pm 0.029$ & $1.762 \pm 0.036$ & $1.219 \pm 0.027$ \\
QW Gem & $0.438 \pm 0.014$ & $1.314 \pm 0.035$ & $0.747 \pm 0.017$ & $1.258 \pm 0.029$ \\
AP Leo & $1.460 \pm 0.042$ & $0.434 \pm 0.017$ & $1.477 \pm 0.045$ & $0.817 \pm 0.025$ \\
V2150 Cyg & $2.350 \pm 0.338$ & $1.885 \pm 0.271$ & $1.982 \pm 0.054$ & $1.786 \pm 0.050$ \\
\hline
\end{tabular}

- may be too conservative rather than overly optimistic in providing estimates of the physical parameter uncertainties.

An extensive discussion of uncertainties of parameters resulting from the DDO spectroscopic program has been presented by Rucinski (2002b). Particular attention was paid to errors for components of very close binary systems with heavily broadened lines. The reader is referred to this publication (especially Figs. 3 and 4) for several statistical data based on the first 60 orbits of this program. While the individual random errors of radial velocities typically range between 2 and $20 \mathrm{~km} \mathrm{~s}^{-1}$, the errors of $K_{i}$ are typically around $1-2 \mathrm{~km} \mathrm{~s}^{-1}$. A brief discussion of the likely systematic errors presented in the same publication suggests that these can be of similar magnitude to random erros, but are much more dificult to characterize.

The absolute parameters of the components for the four systems analysed in this paper are given in Table 3.

We conclude by stating that in this paper we present a rather extensive discussion of the rationale and goals for this type of investigation. While the results for the first four targets are rather extensively discussed, we defer a discussion of the properties of these stars in a broader astrophysical context to a later paper which will summarize the results for several binary systems of this series.

Acknowledgements. This project was supported by the NATO linkage grant No. PST.CLG.978810 and the Polish National Committee grant No. 2 P03D 006 22. The computations were performed at ACK "Cyfronet" in Cracow under grant No. KBN/UJ/015/95, which we gratefully acknowledge.

\section{References}

Abt, H. 1981, ApJS, 243, 894

Bartolini, C., \& Battistini, P. 1969, Publ. Bologna 10, No. 3

Binney, J., Dehnen, W., \& Bertelli, G. 2000, MNRAS, 318, 658
Claret, A., Díaz-Cordovés, J., \& Gimenez, A. 1995, A\&AS, 114, 247

Díaz-Cordovés, J., Claret, A., \& Gimenez, A. 1995, A\&AS, 110, 329 Harmanec, P. 1988, BAC, 39, 329

Kreiner, J. M., Krzesinski, J., Pokrzywka, B., et al. 1993, Proc. IAU Coll. 136, ed. I. Elliot, \& C. J. Buttler, 80

Kreiner, J., Kim C., \& Nha Il-Seong 2001, An Atlas of O-C Diagrams of Eclipsing Binary Stars (Krakow Pedagogical University Press)

Li, L., Zhang, F., \& Han, Z. 2002, PASJ, 54, 73

Lu, W., \& Rucinski, S. M. 1999, AJ, 118, 515

Lu, W., Rucinski, S. M., \& Ogloza W. 2001, AJ, 122, 402

Mauder, H 1967, A\&A, 17, 1

Ogloza, W., Zola, S., Tremko, J, \& Kreiner, J. M. 1998, A\&A, 340, 810

Rahunen, T. 1981, A\&A, 102, 81

Rahunen, T. 1982, A\&A, 109, 66

Rahunen, T. 1983, A\&A, 117, 235

Rasio, F. A. 1995, ApJ, 444, L41

Rucinski, S. M. 1994, PASP, 106, 462

Rucinski, S. M. 1997, AJ, 113, 1112

Rucinski, S. M. 1998, AJ, 116, 2998

Rucinski, S. M. 2000, AJ, 120, 319

Rucinski, S. M. 2002a, PASP, 114, 1124

Rucinski, S. M. 2002b, AJ, 124, 1746

Rucinski, S. M., \& Lu, W. 1999, AJ, 118, 2451

Rucinski, S. M., Lu, W., \& Mochnacki, S. W. 2000, AJ 120, 1133

Rucinski, S. M., Lu W., Mochnacki S. W., Ogloza W., \& Stachowski G. 2001, AJ, 122, 1974

Rucinski, S. M., Lu, W., Capobianco, C. C., et al. 2002, AJ, 124, 1738

Rucinski, S. M., Capobianco, C. C., Lu, W., et al. 2003, AJ, 125, 3258

Strohmeier, W., Knigge, R. 1961, Ver. Bamberg, 5, 10

Vetesnik, M. 1977, BAICz, 28, 120

Vilhu, O. 1981, A\&AS, 78, 401

Vilhu, O. 1982, A\&A, 109, 17

Webbink, R. F. 1976, ApJ, 209, 829

Wilson, R. E., Devinney, E .J. 1973 ApJ, 182, 539

Wilson, R. E. 1979, ApJ, 234, 1054

Wilson, R. E. 1993, Documentation of Eclipsing Binary Computer Model

Zhang, J.-T., Zhang, R.-X., Zhai, D.-S. 1992, ChA\&A, 16, 407 